\title{
Free Space Link Budget Evaluation of UWB-IR Systems
}

\author{
Sathaporn Promwong, Wataru Hachitani, Jun-ichi Takada \\ Graduate School of Science and Engineering, \\ Tokyo Institute of Technology \\ O-okayama Minami 6 Bldg., 2-12-1, O-okayama, Meguro-ku, 152-8552, Tokyo, JAPAN \\ Email: ken@ap.ide.titech.ac.jp
}

\begin{abstract}
Ultra wideband impulse radio (UWB-IR) technology is an ideal candidate for wireless networks that can be utilized for short-range, high-speed, low power, and low cost indoor applications. The link budget of the free space propagation loss is usually estimated by using Friis' transmission formula. However, it is not directly applicable to ultra wideband impulse radio transmission systems, in particular the single band impulse radio, as the formula is expressed as a function of the frequency. Moreover, the waveform may be distorted due to the frequency characteristics of the antenna, and the comparison between $T x$ and $R x$ waveforms is not straightforward. This paper discusses the free space link budget evaluation of UWB-IR systems based on the extended Friis' transmission formula. The matched filter is considered at the receiver side to maximize the SNR for evaluation. An experimental evaluation of the antenna transfer function needs the three-antenna method for the calibration of reference antenna. The technique gives very accurate results and is very useful for design and evaluation of UWB impulse radio transmission systems, especially for the evaluation of waveform distortion effects.
\end{abstract}

Keywords: UWB-IR, link budget, Friis' transmission formula, three-antenna method

\section{INTRODUCTION}

Wireless personal area networks (WPANs) are required to have high data rate, low power consumption, and low cost. The ultra wideband (UWB) radio technology is an ideal candidate focusing on wireless PAN [1], [2].

In UWB communication systems, the antennas are significantly pulse-shaping filters. Any distortion of the signal in the frequency domain causes the distortion of the transmitting pulse shape. Consequently this will increase the complexity of the detection mechanism at the receiver [3]. Moreover, low cost, geometrically small and still efficient structures are required for typical wireless applications. Therefore the antenna design for UWB signal radiation is one of the main challenges [4], [5].

Even if the channel is in line of sight (LOS), Friis' transmission formula cannot be directly applied to the UWB radio as the bandwidth of the pulse is extremely wide. Furthermore, simple comparison between waveforms of transmitter and receiver is not significant because of the distortion of the waveform caused by the frequency response of the antenna.

In this paper, we discuss the free space link budget evaluation of UWB-IR systems. This scheme is based on the Friis' transmission formula, adapted for UWB, in the sense that we would like to derive the equivalent antenna gain for UWB systems. The transmission waveform and the matched filter reception are keys for the extension of the Friis' formula to UWB. To know the antenna transfer function by measurement, we need the threeantenna method for calibration of the reference antenna. An experiment is carried out using the biconical antenna for UWB operation in the anechoic chamber.

\section{EXTENSION OF FRIIS' TRANSMISSION FORMULA FOR UWB TRANSMISSION SYSTEM}

In this study, we focus on the link budget evaluation of UWB-IR system in free space.

In narrowband systems, the link budget of the free space propagation loss is usually estimated by using Friis' transmission formula [6]. However, it is not directly applicable to the UWB-IR transmission system, as the formula is expressed as a function of the frequency. Moreover, the waveform may be distorted due to the frequency characteristics of the antenna. Ref. [7] treats the special cases of the constant gain and the constant aperture, but no general discussion had been made although it suggested the use of the time-domain antenna effective length.

The Friis' transmission formula [6] has been widely used, and can be applied to the calculation of these LOS channels.

$$
G_{\text {Friis }}(f)=\frac{P_{\mathrm{r}}(f)}{P_{\mathrm{t}}(f)}=G_{\mathrm{f}}(f) G_{\mathrm{r}}(f) G_{\mathrm{t}}(f),
$$

where $G_{\mathrm{r}}$ and $G_{\mathrm{t}}$ are Rx and Tx antenna gain,

$$
G_{\mathrm{f}}(f)=\left(\frac{\lambda}{4 \pi d}\right)^{2}
$$

is the free space propagation gain (less than unity in practice), $\lambda=\frac{c}{f}$ is the wavelength, $c$ is the velocity of the 


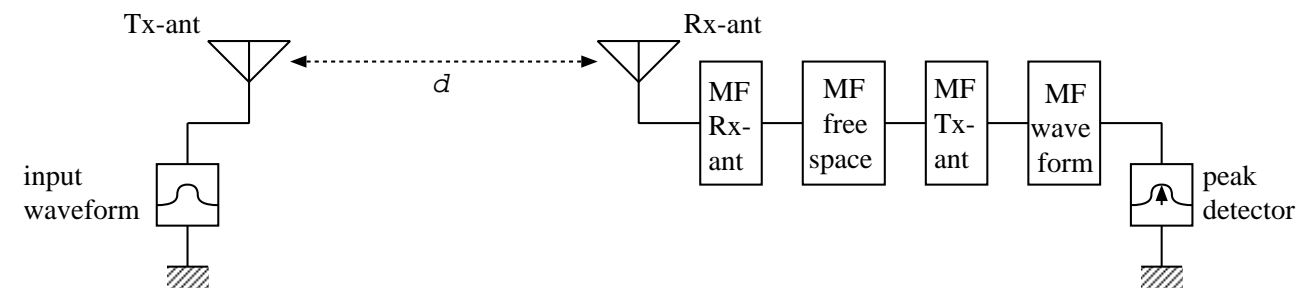

Fig. 1. Block diagram of transmission system for the extension of Friis' transmission formula to treat UWB signal.

light, $f$ is the operating frequency, and $d$ is the separation between transmitter and receiver antennas.

It is noted, however, that Eq. (1) is satisfied only at some certain frequency, and is not directly applicable to UWB systems. The Friis' transmission formula shall be extended to take into account the transmission signal waveform and its distortion as well [8], [9].

Input signal $v_{\mathrm{i}}(t)$ at the transmitter port is expressed as the convolution of an impulse input and the pulse shaping filter $h_{\mathrm{i}}(t)$ as

$$
v_{\mathrm{i}}(t)=E_{\mathrm{i}} \delta(t) * h_{\mathrm{i}}(t),
$$

where

$$
\int_{-\infty}^{\infty} h_{\mathrm{i}}^{2}(t) \mathrm{d} t=\int_{-\infty}^{\infty}\left|H_{\mathrm{i}}(f)\right|^{2} \mathrm{~d} f=1 .
$$

Friis' formula is extended taking into account the transmission waveform as

$$
H_{\mathrm{e}-\text { Friis }}(f)=\frac{V_{\mathrm{r}}(f)}{E_{\mathrm{i}}}=H_{\mathrm{f}} H_{\mathrm{i}} \mathbf{H}_{\mathrm{r}} \cdot \mathbf{H}_{\mathrm{t}},
$$

where

$$
\begin{aligned}
\mathbf{H}_{a} & =\mathbf{H}_{a}\left(\theta_{a}, \varphi_{a}, f\right) \\
& =\hat{\boldsymbol{\theta}}_{a} H_{a \theta}\left(\theta_{a}, \varphi_{a}, f\right)+\hat{\boldsymbol{\varphi}}_{a} H_{a \varphi}\left(\theta_{a}, \varphi_{a}, f\right)(6) \\
a & =\mathrm{r} \text { or } \mathrm{t}
\end{aligned}
$$

is a complex transfer function vector of the antenna relative to the isotropic antenna,

$$
H_{\mathrm{f}}=\frac{\lambda}{4 \pi d} \exp (-\mathrm{j} k d),
$$

is the free space transfer function where

$$
k=\frac{2 \pi}{\lambda}
$$

is the propagation constant. Unit vectors $\hat{\boldsymbol{\theta}}_{\mathrm{a}}, \hat{\boldsymbol{\varphi}}_{\mathrm{a}}$ express the polarization and are defined with respect to the local polar coordinates of each of the antennas. The following relations can be easily derived.

$$
\begin{array}{r}
\hat{\boldsymbol{\theta}}_{\mathrm{r}}=\hat{\boldsymbol{\theta}}_{\mathrm{t}}, \\
\hat{\boldsymbol{\varphi}}_{\mathrm{r}}=-\hat{\boldsymbol{\varphi}}_{\mathrm{t}} .
\end{array}
$$

At the receiver, the matched filter $H_{\mathrm{MF}}(f)$ is introduced to maximize the signal-to-noise ratio (SNR) of the receiver output, as shown in Figure 1.

$$
H_{\mathrm{MF}}(f)=\frac{H_{\mathrm{e}-\text { Friis }}^{*}(f)}{\sqrt{\int_{-\infty}^{\infty}\left|H_{\mathrm{e}-\text { Friis }}(f)\right|^{2} \mathrm{~d} f}},
$$

which satisfies the following constant noise output power condition

$$
\int_{-\infty}^{\infty}\left|H_{\mathrm{MF}}(f)\right|^{2} \mathrm{~d} f=1 .
$$

In this case, the output waveform when $E_{\mathrm{i}}=1$, and the spectrum of the receiver output are $h_{\mathrm{e}-\text { Friis }}(t)$ and $H_{\mathrm{e}-\text { Friis }}(f)$, respectively. The waveform of the output from the matched filter $v_{\mathrm{MF}}(t)$ and the spectrum of the output from the matched filter $V_{\mathrm{MF}}(f)$ are

$$
\begin{aligned}
v_{\mathrm{MF}}(t) & =h_{\mathrm{e}-\text { Friis }}(t) * h_{\mathrm{MF}}(t) \\
& =\frac{h_{\mathrm{e}-\text { Friis }}(t) * h_{\mathrm{e}-\text { Friis }}(-t)}{\sqrt{\int_{-\infty}^{\infty} h_{\mathrm{e}-\text { Friis }}^{2}(t) \mathrm{d} t}} \\
V_{\mathrm{MF}}(f) & =H_{\mathrm{e}-\text { Friis }}(f) H_{\mathrm{MF}}(f) \\
& =\frac{\left|H_{\mathrm{e}-\text { Friis }}(f)\right|^{2}}{\sqrt{\int_{-\infty}^{\infty}\left|H_{\mathrm{e}-\text { Friis }}(f)\right|^{2} \mathrm{~d} f}},
\end{aligned}
$$

taking its maximum as

$$
\begin{aligned}
\max _{t} v_{\mathrm{MF}}(t) & =\int_{-\infty}^{\infty} V_{\mathrm{MF}}(f) \mathrm{d} f \\
& =\sqrt{\int_{-\infty}^{\infty}\left|H_{\mathrm{e}-\text { Friis }}(f)\right|^{2} \mathrm{~d} f .}
\end{aligned}
$$

Equation (15) is the UWB extension of Friis' transmission formula. It includes three elements, namely the frequency characteristics of the antennas, the frequency characteristics of free space propagation, and the spectrum of the transmit signal. It is clear from Eq. (15) that the transmission gain of the UWB signal can not be defined as the product of gains of antennas and a free space channel as Friis' formula (1). Instead, the total transmission gain 
including the effect of the waveform can be obtained as Eq. (15). For the normalization, the reference isotropic antenna with $H_{\text {Iso }}(f)=1$ is considered. The UWB transmission gain can be defined as

$$
G_{\mathrm{UWB}}=\max _{t} v_{\mathrm{MF}}(t) / \max _{t} v_{\mathrm{MF}, \mathrm{Iso}}(t) .
$$

\section{Measurement of Antenna Transfer FUNCTION}

\section{A. Measurement Scheme}

By using the vector network analyzer (VNA), complex transfer functions can be measured. However, this transfer function is a product of transfer functions of Tx and Rx antennas as well as the free space channel. Among them, the free space transfer function is calculated from the distance between the antennas by using Eq. (7). To know the transfer function of the antenna under test (AUT) at the Rx side, the transfer function of the Tx antenna, which is usually a standard antenna, shall be known in advance as the calibration data. The overall measurement scheme is summarized as follows:

Step 1)Calibration of the standard antenna

The standard antenna is calibrated by using the three-antenna method. The three-antenna method has originally been proposed for the measurements of the complex antenna factor [10]. In this method, three linearly-polarized antennas are required, but they do not have to be identical to one another. Three sets of measurements are performed using all combinations of the three antennas pointing toward the same directions as shown in Fig. 2. The result is a set of three simultaneous equations of the form

$$
\begin{aligned}
& S_{21}(f)=H_{1}(f) H_{\mathrm{f}}(f, d) H_{2}(f), \\
& S_{13}(f)=H_{3}(f) H_{\mathrm{f}}(f, d) H_{1}(f), \\
& S_{32}(f)=H_{2}(f) H_{\mathrm{f}}(f, d) H_{3}(f),
\end{aligned}
$$

where $H_{i}(f)$ is the complex frequency transfer function of antenna $i, S_{i j}$ is the measurement result by using Tx antenna $i$ and Rx antenna $j, d$ is the distance between antennas, and $H_{\mathrm{f}}(f, d)$ is the complex transfer function of free space given in Eq. (7). Then, we can estimate the complex frequency transfer function of the antennas by using these equations

$$
\begin{aligned}
H_{1}(f) & =\sqrt{\frac{S_{21}(f) S_{32}(f)}{S_{13}(f) H_{\mathrm{f}}(f, d)}}, \\
H_{2}(f) & =\sqrt{\frac{S_{21}(f) S_{13}(f)}{S_{32}(f) H_{\mathrm{f}}(f, d)}}, \\
H_{3}(f) & =\sqrt{\frac{S_{32}(f) S_{13}(f)}{S_{21}(f) H_{\mathrm{f}}(f, d)}} .
\end{aligned}
$$

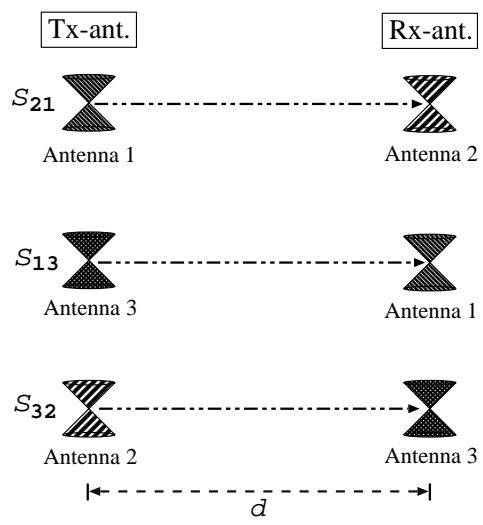

Fig. 2. Three antenna model.

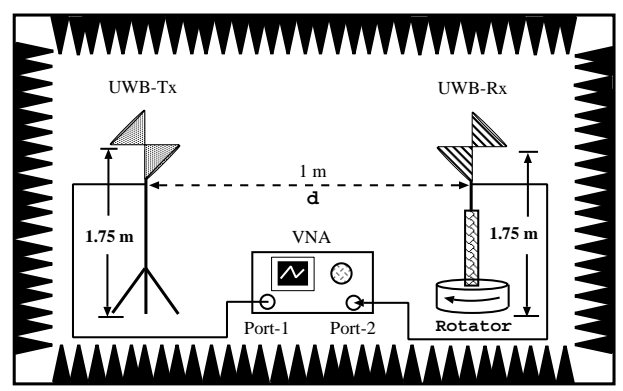

Fig. 3. The instrument setup.

Step 2)The transfer function of AUT is measured.

By using the standard antenna and the AUT as Tx and Rx antennas respectively, the transfer function between $\mathrm{Tx}$ and $\mathrm{Rx}$ antenna ports is expressed as

$$
S_{21}(f)=H_{\mathrm{AUT}}(\theta, \varphi, f) H_{\mathrm{f}}(f, d) H_{\mathrm{Std}}(f),
$$

and the transfer function of AUT is obtained by

$$
H_{\mathrm{AUT}}(\theta, \varphi, f)=\frac{S_{21}(f)}{H_{\mathrm{f}}(f, d) H_{\mathrm{Std}}(f)} .
$$

\section{B. Experimental Setup and Measurement Model}

The VNA was operated in the response measurement mode, where Port-1 was the transmitter port (Tx) and Port-2 was the receiver port $(\mathrm{Rx})$, respectively. Biconical antennas with the maximum diameter of $65.3 \mathrm{~mm}$ and the length of $37 \mathrm{~mm}$ are used both as the standard antennas and as AUT [11]. The measurement was done in the anechoic chamber. Both $\mathrm{Tx}$ and $\mathrm{Rx}$ antennas were fixed at the height of $1.75 \mathrm{~m}$ and separated at a distance of $1 \mathrm{~m}$. The setup is sketched in Fig. 3 .

Figure 4 shows the orientations of the $S_{21}$, transfer function measurement for $\mathrm{Tx}$ and $\mathrm{Rx}$ antennas. The $\mathrm{Tx}$ antenna is fixed at pointing angle $0^{\circ}$ and the Rx antenna is rotated from pointing angle $0^{\circ}$ to $360^{\circ}$ with each step at $5^{\circ}$. 


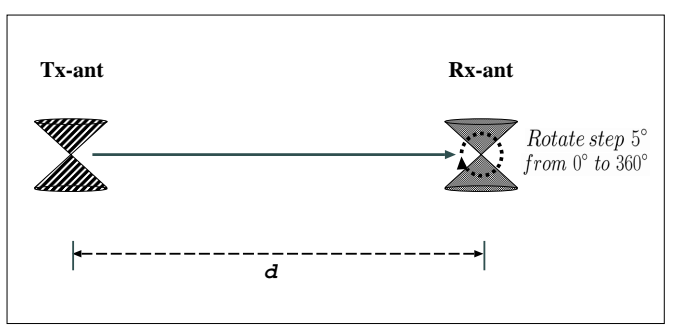

Fig. 4. Top view antenna setting.

TABLE I

EXPERIMENTAL SETUP PARAMETERS.

\begin{tabular}{lc}
\hline Parameter & Value \\
\hline Frequency range & $3 \mathrm{GHz}$ to $11 \mathrm{GHz}$ \\
Number of frequency points & 1601 \\
Dynamic power range & $80 \mathrm{~dB}$ \\
Tx antenna height & $1.75 \mathrm{~m}$ \\
Rx antenna height & $1.75 \mathrm{~m}$ \\
Distance between Tx and $\mathrm{Rx}$ & $1 \mathrm{~m}$ \\
Rx rotate range & $0^{\circ}$ to $360^{\circ}$ \\
Rx rotate step & $5^{\circ}$ \\
\hline
\end{tabular}

\section{Parameters of Experiment and Calibration Techniques}

The important parameters for the experiments are listed in Table I.

It is noted that the calibration is done at the connectors of the cables to be connected to the antennas. Therefore, all the impairments of the antenna characteristics are included in the measured results.

\section{UWB waveform Transmission}

The effect of the waveform distortion is more obvious when the bandwidth is wider. We considered the impulse radio signal that fully covers the FCC band [12], i.e., $3.1 \sim 10.6 \mathrm{GHz}$. The center frequency and the bandwidth were therefore set to be $f_{0}=6.85 \mathrm{GHz}$ and $f_{\mathrm{b}}=$ $7.5 \mathrm{GHz}$, respectively. The transmit waveform assumed in the simulation was a single ASK pulse with the carrier frequency $f_{0}$. To satisfy the bandwidth requirement of $f_{\mathrm{b}}$, the pulse length was set to be $\frac{2}{f_{\mathrm{b}}}$. Then the signal was band-limited by a Nyquist roll-off filter with rolloff factor $\alpha=0$ (rectangular window) and passband $\left(f_{0}-\frac{f_{\mathrm{b}}}{2}, f_{0}+\frac{f_{\mathrm{b}}}{2}\right)$. Figure 5 shows the transmit pulse waveform. The transmission process of the pulse waveform is simulated based on the measured transfer function of the antenna.

\section{EXAMPLE Result AND Discussion}

Figure 6 shows the magnitude of the measured antenna transfer function and its phase is also shown in Fig. 7. We can particularly see the frequency characteristic of the antenna transfer function at each pointing angle. As the

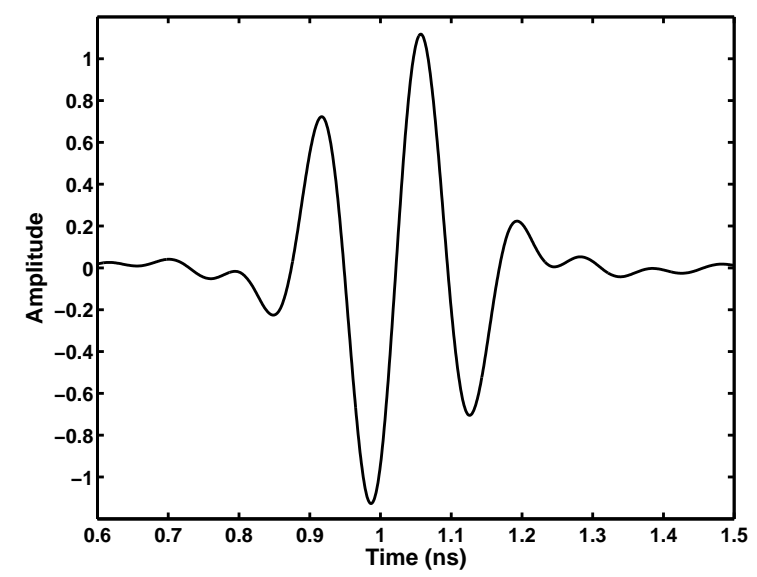

Fig. 5. The transmission waveform of UWB signal.

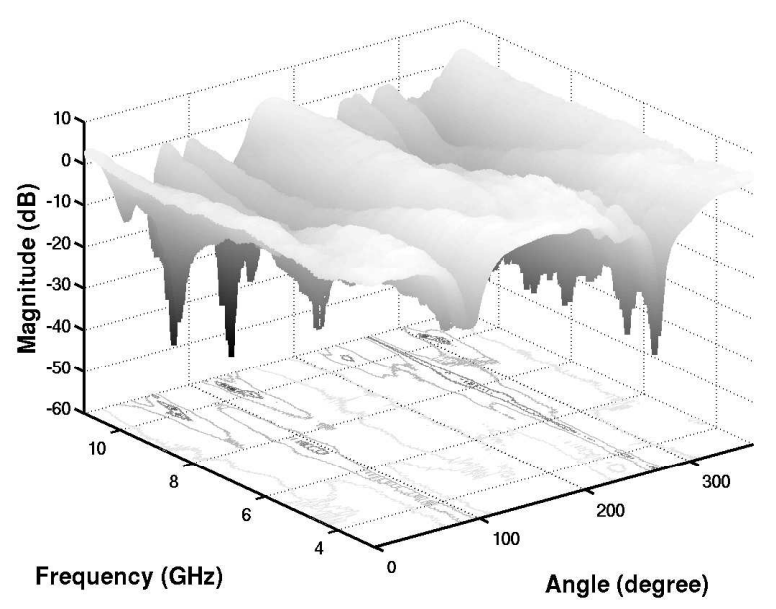

Fig. 6. Antenna transfer function: magnitude.

AUT is the broadband biconical antenna, the ideal linear phase is almost realized, except for the null directions which change by the frequency.

The UWB signal shown in Fig. 5 is used as the transmission waveform. The received waveforms at the output of the matched filters is evaluated. The relative gain is defined as Eq. (16). In practice, it is quite complicated and is not feasible to implement the adaptive matched filter to adjust for the antennas. Therefore, the matched filter designed for an isotropic antenna is also considered and is compared with the ideal matched filter. Specifically, Eq. (14) is replaced by

$$
H_{\mathrm{MF}, \text { Iso }}(f)=\frac{H_{\mathrm{e}-\text { Friis, Iso }}^{*}(f)}{\sqrt{\int_{-\infty}^{\infty}\left|H_{\mathrm{e}-\text { Friis, Iso }}(f)\right|^{2} \mathrm{~d} f}},
$$

where

$$
H_{\mathrm{e}-\text { Friis, Iso }}(f)=H_{\mathrm{f}}(f) H_{\mathrm{i}}(f)
$$




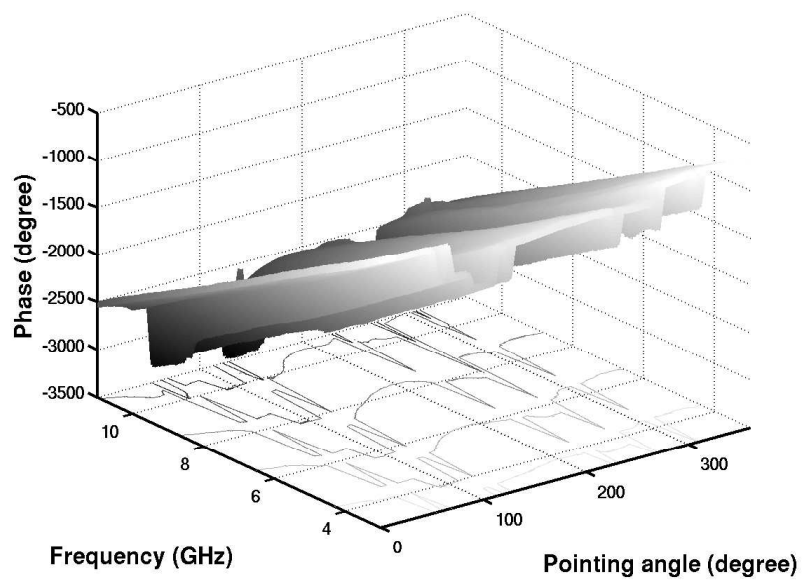

Fig. 7. Antenna transfer function: phase.

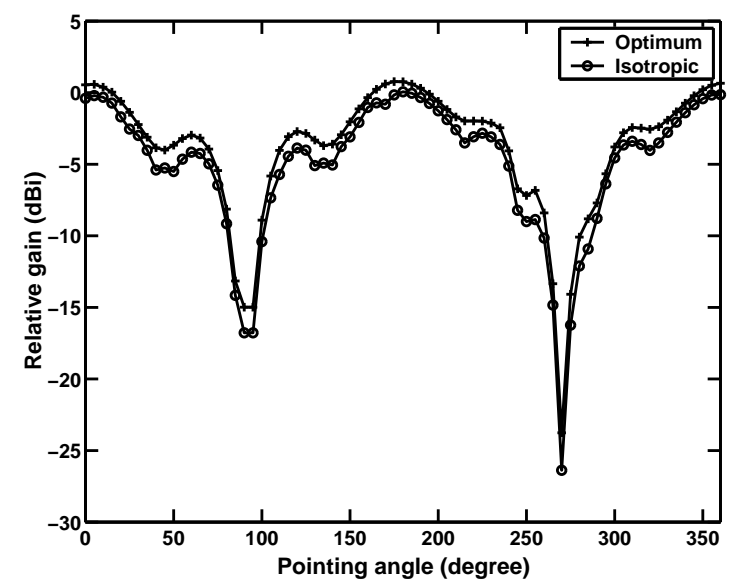

Fig. 8. UWB transmission gain.

Figure 8 shows the UWB transmission gain versus antenna pointing angle that uses the optimum matched filter compared with the matched filter for an isotropic antenna. The peaks are found at $0^{\circ}, 180^{\circ}$, and $360^{\circ}$ pointing angles which corresponds to the broadside of the antenna. The nulls are observed at $90^{\circ}$ and $270^{\circ}$ pointing angles.

Table II summarizes the UWB transmission gain by using Eq. (16), in comparison with the IEEE 802.15.3a path loss model which uses the Friis' formula with geometric average frequency [13]. Since the biconical antnena has linear phase property, the ideal IEEE 802.15.3a and the isotropic matched filter are not significanty different.

\section{CONCLUSION}

This paper presented the UWB transmission gain, which is an extension of Friis' transmission formula in order to take into account the transmit waveform and the matched filter into the system for the free space link bud-
TABLE II

COMPARISON OF THE UWB TRANSMISSION GAIN FOR BICONICAL ANTENNAS POINTING THE MAIN BEAMS TO EACH OTHER.

\begin{tabular}{lr}
\hline Method & Gain [dBi] \\
\hline Optimum MF & -2.93 \\
Isotropic MF & -3.97 \\
IEEE 802.15.3a [13] & -2.26 \\
\hline
\end{tabular}

get evaluation of UWB-IR. The experimental examples using the biconical antennas are presented. Our method proposed for UWB transmission and the measurement results were found to be close to the IEEE $802.15 .3 a$ path loss model.

\section{ACKNOWLEDGEMENT}

The authors would like to thank Mr. Kimio Sakurai, Dr. Ichirou Ida, Mr. Gilbert S. Ching, Mr. Katsuyuki Haneda, and Ms. Navarat Lertsirisopon, all from Tokyo Institute of Technology, for their help in the experiments and review of this paper. This research is partly supported by the fund from the Telecommunications Advancement Organization (TAO) of Japan.

\section{REFERENCES}

[1] K. Siwiak, "Ultra-Wide Radio: Introduccing a New Technology," 2001 Spring IEEE Veh. Tech. Conf. (VTC), Plenary session, May 2001.

[2] K. Siwiak, "Ultra-Wide Radio: The emergence of an Important RF Technology", Proc. 2001 Spring IEEE Veh. Tech. Conf. (VTC), May 2001.

[3] K. Siwiak, "Impact of UWB Transmission on Generic Receiver," Proc. 2001 Spring IEEE Veh. Tech. Conf. (VTC), May 2001.

[4] H.F. Harmuth and S. Ding-Rong, "Antennas for Nonsinusiodal Wave - Part I: Radiators," IEEE Trans. Elec. Mag. Compat. vol. EMC-25, no. 1, pp. 13-24, Feb. 1983.

[5] H.F. Harmuth and S. Ding-Rong, "Antennas for Nonsinusiodal Wave - Part II: Sensors," IEEE Trans. on Elec. Mag. Compat., vol. EMC-25, no. 1, no. 2, pp. 107-115, May 1983.

[6] H.T. Friis, "A Note on a Simple Transmission Formula," Proc. IRE vol. 34, no. 5, pp. 254-256, May 1946.

[7] United States of America, "Path Loss Calculations for UltraWideband Signals in Indoor Environments," ITU-R Document 3K/30-E, pp. 1-14, Nov. 2003.

[8] J. Takada, S. Promwong and W. Hachitani, "Extension of Friis Transmission Formula for Ultra-Wideband Systems," IEICE Tech. Rep., WBS2003-8/MW2003-20, May 2003.

[9] A.H. Mohammadian, A. Rajkotia, and S.S. Soliman, "Characterization of UWB Transmit-Receive Antenna System," Proc. IEEE Conf. Ultra Wideband Syst. Tech. (UWBST) 2003, Nov. 2003.

[10] S. Ishigami, H. Iida and T. Iwasaki, "Measurements of Complex Antenna Factor by the Near-Field 3-Antenna Method," IEEE Trans. Electromagnetic Com., vol. 38, no. 3, pp. 424-432, July 1992

[11] S. Promwong, W. Hachitani, J. Takada, P. Supanakoon, and P Tangtisanon "Experimental Study of Ultra-Wideband Transmission Based on Friis' Transmission Formula," The Third International Symposium on Communications and Information Technology (ISCIT) 2003, vol. 1. pp. 467-470, Sept. 2003.

12] Federal Communications Commission, "Revision of Part 15 of the Commission's Rules Regarding Ultra-Wideband Transmission Systems," First Report and Order, FCC 02-48, Apr. 2002.

[13] J. Foerster, "Channel Modeling Sub-committee Report Final," IEEE P802.15-02/368r5-SG3a, Nov. 2002. 